\title{
Autoestima socioprofesional del Maestro Rural
}

Juan José Sáchez de Horcajo

Presentamos en estas páginas algunos de los resultados parciales de la investigación sociológica, realizada en el Instituto de Ciencias de la Educación de la UUniversidad Complutense sobre: "El profesorado rural de la Educación General Básica en Castilla y León".

El estudio pretende analizar entre otras variables sociolgicas, el grado de integración rural, el nivel de profesionalización, el prestigio y autoestima socioprofesional, el conservadurismo y permisividad, la socialización profesional, actitudes sociopolíticas y religiosidad.

La metodología empleada para la realización del informe se ha fundamentado sobre la combinación de técnicas cuantitativas y cualitativas de obtención y análisis de datos: encuesta a una muestra representativa de 630 maestros, grupos de discusión con distintos colectivos de maestros de la región y entrevistas personales de casos "típicos ideales".

Referimos aquí algunos resultados relativos a la autoestima socioprofesional del maestro rural en dicha región (1).

\section{1.- El concepto de autoestima}

Entendemos por autoestima la evaluación que cada individuo hace ordinariamente de sí mismo. Expresa una actitud de aprobación o desaprobación, e indica la amplitud de cómo cada sujeto cree ser capaz de obrar, tener éxito, tener significación, etc... En pocas palabras, la autoestima es un juicio personal de valía, que se expresa en las actitudes que cada uno tiene de sí mismo. Es una experiencia subjetiva que el individuo proyecta sobre los otros veladamente y como expresión de su conducta.

La autoestima puede ser pensada de manera multidimensional, según la observación o posición individual sobre un amplio número de dimensiones, como cultura, atractivo físico, honestidad, inteligencia, trabajo... etc. Cada una de ellas puede ser tomada en una escala evaluativa, de acuerdo como cada

(1) Utilizaremos indistintamente los términos de profesor de E.G.B. o maestro, dada la identificación comúnmente atribuida. 
individuo juzga su valía en cada dimensión. La autoestima global vendría dada por la media de todas las evaluaciones.

El esquema sugiere inmediatamente problemas a la investigación y medida, pues depende del tipo de idiosincrasia, tipo de medidas y dimensiones, etc. También en este campo, como en otras categorizaciones, en sociología o psicología, se intenta la construcción de un "tipo ideal" (2).

Las escalas de medida de la autoestima - "self-steem" o "self-concept"son muy variadas; quizás hay más de doscientas, muy difíciles de precisar y considerar.

En nuestro estudio, para medir la autoestima y autoprestigio comparativo del profesorado rural preguntamos a los maestros sobre tres aspectos profesionales: prestigio social atribuido, capacitación o competencia profesional y remuneración económica profesional, para que dentro de una escala númeríca bipolar de 0 a 9 puntuaran comparativamente su propia profesión y una serie de profesiones equiparables.

Analicemos, brevemente y por separado, cada uno de estos indicadores.

\section{2.- Prestigio social interprofesional}

Es bien patente cómo en las sociedades modernas el status social y categoría profesional tiene comúnmente atribuido un nivel de prestigio social. Por otra parte, aunque no presenta una rigurosa coincidencia, se observa con frecuencia un cierto paralelismo entre el prestigio social atribuido y la remuneración económica que obtiene cada profesión.

No vamos a entrar aquí en las cuestiones epistemológicas sobre la atribución de status y de prestigio social o sobre la configuración de los estratos o categorías. Anotamos simplemente cómo en las sociedades modernas algunas profesiones se encuentran entre las ocupaciones más prestigiosas y mejor remuneradas. Los estudios sobre prestigio social en Gran Bretaña y Estados Unidos han demostrado de forma consistente, que las profesiones más antiguas son todavía consideradas como de status más elevados, aunque algunas profesiones más modernas han adquirido un prestigio social muy relevante.

L'a condición social de enseñante siempre ha intentado escapar, más o menos parcialmente, a todo intento de valoración comparada, en el establecimiento de categorías.

En el estudio realizado por el INEM, en el año 1978, sobre el prestigio social de las ocupaciones en España, se analiza el prestigio comparativo de $\mathbf{4 0}$ ocupaciones, entre ellas la de maestro. Este estudio constata el relativamente alto grado de prestigio que tienen en nuestro país los profesores de básica en

(2) Véase COOPERSMITH, S.: "The antecedents of self-steem", Freedman, San Francis$\infty$, 1967. SHERWOOD, J.J.: "Self-identity and self-actualization: a theory and research". Universidad de Michigan, 1962

Véase también el concepto de "personal competence" en CAMPBELL, A.: The American Voter, Nueva York, Wiley, 1960. 
las Zonas rurales, y en las Zonas semi-urbanas; a medida que el estrato de población disminuye, el grado de prestigio de este grupo aumenta. El maestro aparece situado en términos de prestigio por encima de estas ocupaciones (de mayor prestigio): oficial administrativo, empleado de banca, mecanógrafa, reparador de electrodomésticos, capataz de obra... etc. Por encima del maestro figuraban (en orden creciente de prestigio): funcionario medio, dueño de tienda media, sacerdote, periodista, etc... El grado de prestigio acordado al maestro correlaciona positivamente con la edad de los encuestados. La correlación es inversa con las variables de nivel de estudios, estrato de población y nivel de ingresos (3).

En nuestra investigación tratamos de constatar la opinión del profesorado rural respecto al prestigio social atribuido a distintos colectivos profesionales. Que el maestro haga de portavoz de la conciencia colectiva. Desde la percepción personal del prestigio atribuido se puede también percibir la autoestima del prestigio profesional. Veamos las aportaciones más significativas.

Pedimos a los maestros que evaluasen el prestigio social atribuido a diversos profesionales, puntuando de 0 a 9; y clasificamos las puntuaciones en tres grupos: baja, 0 a 3 puntos; media, 4 a 6 puntos; alta, 7 a 9 puntos. La lista de profesiones y su evaluación se refleja en la tabla siguiente:

Tabla I. Prestigio social atribuido a las profesiones

\section{PROFESION}

Médico ...............

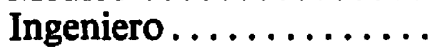

Militar...............

Empresario ............

Sacerdote ............

Juez

Diputado

Arquitecto............

Abogado

Profesor

Agricultor ............
Puntuación ALTA MEDIA

\section{BAJA}

7,6

4,4

22,1

15,6

19,7

10,0

21,2

5,5

10,0

11,1

20,1
No contesta

11,2

14,3

14,9

15,5

12,4

13,5

14,8

13,9

14,4

10,4

14,1 total

100,0

100,0

100,0

100,0

100,0

100,0

100,0

100,0

100,0

100,0

100,0

Las profesiones con mayor prestigio entre los profesores son las siguientes: Médico $(67,4 \%)$, Ingeniero $(62 \%)$, Arquitecto $(55,9 \%)$, Juez $(51 \%)$, a continuación viene la propia profesión del Profesor $(47,6 \%)$. Todas estas profesiones tienen la puntuación alta señalada por más de la mitad de los profesores que contestan.

(3) INEM: El prestigio social de las ocupaciones en Espana. Madrid, 1978. 
Los profesores sitúan la propia profesión por encima de las siguientes profesiones: Sacerdote (40\%), Abogado (39,8\%), Diputado (33,9\%). Las profesiones con menor prestigio entre los profesores son: Empresario, Agricultor y Militar. Esta escala ha sido realizada fijándose en la escala alta y completándola con la baja.

A todo lo anterior hay que añadir que el porcentaje de no contesta es alto. Ello nos ha de hacer ponderar con cautela las puntuaciones.

La propia profesión aparece bien posicionada en la escala de prestigio que los propios profesores señalan. Así aparece entre las profesiones de Juez y de Sacerdote. Es de notar que la posición de la profesión de Militar es la más baja; es decir, es la que menos prestigio recibe de los profesores que contestan.

Podemos hacer notar que existen interrelaciones entre la escala de prestigio y el sexo de los profesores. Sefialamos las más significativas.

1.-Profesiones que tienen mayor prestigio para las mujeres que para los varones:

- Profesor. Las mujeres dan mayor puntuación de prestigio que los varones. Así por ejemplo, hay un $\mathbf{9 , 4 \%}$ más de mujeres que de varones que dan puntuación alta.

- Sacerdote. Hay 9,9\% más de varones que de mujeres que dan puntuación media. También hay un $3,4 \%$ más de varones que de mujeres que dan puntuación baja.

- Agricultor. Así por ejemplo, hay un 4,8\% más de mujeres que de varones que dan puntuación alta.

2. Profesiones que tienen menor prestigio para las varones que para las mujeres (igualan porcentajes de puntuación alta, difieren significativamente en las restantes)

- Abogado. Por ejemplo, hay un $7 \%$ más de varones que de mujeres que dan puntuación baja.

3. - Profesiones que tienen mayor prestigio para los varones que para las mujeres.

- Arquitecto. Por ejemplo, hay un 8,5\% más de varones que de mujeres que dan puntuación alta.

Existen también correlaciones respecto a la edad. El análisis podemos resumirlo como sigue:

1.-Correlaciones que se dan en ambos sexos:

- Sacerdote. A mayor edad del profesorado, mayor puntuación de prestigio hacia dicha profesión. Asi por ejemplo, hay un $13,6 \%$ más de mujeres entre 36 y 50 afos que dan puntuación alta.

2.- Correlaciones que sólo se dan en varones:

- Médico. A mayor edad, menor puntuación. Son los más jóvenes quienes le dan mayor puntuación. Por ejemplo, hay un $24,3 \%$ más de varones entre 20 y 35 años que de varones entre 51 y 70 años que dan puntuación de prestigio alta.

- Diputado. A mayor edad, menor puntuación. Los más jóvenes son 
- Abogado. A mayor edad, menor puntuación de prestigio. Por ejemplo, hay un $13 \%$ más de varones entre 20 y 35 años que de varones entre 36 y 50 años que dan puntuación alta.

- Profesor. A mayor edad, mayor puntuación de prestigio. Por ejemplo, hay un $6,2 \%$ más de varones entre 36 y 50 años que de varones entre 20 y 35 años que dan puntuación $a l$ ta.

3.- Sólo se dan en mujeres.

- Empresario. A mayor edad, mayor puntuación de prestigio. Por ejemplo, hay un $12,3 \%$ más dé mujeres entre 51 y 70 años que de mujeres entre 36 y 50 años que dan puntuación alta.

Algunas variaciones importantes que aparecen en las opiniones de los maestros rurales respecto al prestigio interprofesional, estiman que el cambio de denominación de "maestro" por el de "profesor" ha repercutido en la atribución de su prestigio social. Esta variación es cosiderada como positiva por parte del profesorado más joven; sin embargo, los profesores de mayor edad consideran que el cambio ha repercutido negativamente en la atribución de su prestigio social y profesional.

El cambio de denominación ha llevado consigo, sin duda, una pérdida de la identidad del oficio de maestro. El rol y función de profesor especialista, como manifestación del tecnicismo académico, no parece fácilmente asumible por el medio rural.

Otro factor que condiciona, según los propios profesores, su prestigio en el medio rural, es la residencia en permanencia del maestro en el pueblo. La mera residencia en el pueblo asigna al maestro un carácter de notabilidad y asignación de unas funciones reconocidas de vigilancia y protección de la infancia, animador y representante de la educación, la cultura y la "urbanidad", intermediario y gestor de la burocracia y la Administración, censor rutinario del comportamiento social de la juventud. La pérdida de estos y otros papeles atribuidos difusamente al maestro residente en permanencia en el pueblo, ha devaluado considerablemente la estima y el prestigio social del maestro en el medio rural.

\section{3.- Retribución profesional}

Aunque no presenta una rigurosa coincidencia, se observa, comuńmente, un cierto paralelismo entre el prestigio social atribuido y la remuneración económica que obtiene cada profesión. Los profesionales con status social más elevado mantienen todavia la media de ingresos más altos. En el contexto de 
una sociedad crematística y materializada, éste fenómeno podría expresarse vulgarmente con el refrán: "Dime cuanto ganas y te diré quién eres".

En el desarrollo de nuestras entrevistas con los maestros, esta conexión entre el nivel de retribuciones económicas y nivel de prestigio social es comúnmente asumida y manifestada por ellos. Consideran, conforme a la opinión común, que el prestigio social atribuido a cada profesión corre parejo con la cuantía de la retribución percibida por el ejercicio de la misma. Algunos dichos populares respecto a los maestros, y repetidos por ellos mismos en las entrevistas, son muy expresivos de la autovaloración: "El maestro rural ha pasado de ser compadecido y admirado a ser envidiado". "Se ha trastocado el dicho popular: "pasas más hambre que un maestro-escuela", por el de "vives mejor que un maestro-escuela".

La evolución de los sueldos de los maestros nacionales en las últimas décadas es enormemente expresiva de este fenómeno (4).

Tabla II. Evolución de los sueldos del Magisterio

$\begin{array}{ccc}\text { AÑOS } & \begin{array}{c}\text { HABERES MAXIMOS } \\ \text { MENSUALES }\end{array} & \begin{array}{c}\text { HABERES MINIMOS } \\ \text { MENSUALES }\end{array} \\ 1943 & 1.100 & 333,33 \\ 1951 & 1.560 & 780 \\ 1962 & 2.690 & 1.400 \\ 1973 & 20.000 & 12.000 \\ 1975 & 25.790 & 16.390 \\ 1982 & 78.350 & 48.240\end{array}$

Veamos en la tabla siguiente la estimación comparativa que tienen los maestros con relación a las remuneraciónes percibidas por otros profesionales equiparables. La pregunta se planteaba en estos términos: ¿Respecto a lo que ganan o cobran estos profesionales, en relación con lo que hacen, cree Vd. que cobran demasiado, lo justo, poco?

Tabla III. Opinión sobre la retribución interprofesional

$\begin{array}{lccccc}\text { Profesión } & \text { Demasiado } & \text { Justo } & \text { Poco } & \text { No Contesta } & \text { Total } \\ \text { Médico ....... } & 66,6 & 12,8 & 1,5 & 17,9 & 100,0 \\ \text { Ingeniero ..... } & 54,3 & 22,3 & 0,9 & 22,5 & 100,0 \\ \text { Militar........ } & 59,1 & 20,2 & 0,8 & 19,9 & 100,0 \\ \text { Empresario.... } & 59,4 & 13,7 & 3,3 & 23,6 & 100,0 \\ \text { Sacerdote ..... } & 7,6 & 16,4 & 58,0 & 18,0 & 100,0\end{array}$

(4) Son salarios brutos, descuentos 21,6\%. Fuente Anuario del Magisterio. 


$\begin{array}{lrrrrr}\text { Juez .......... } & 33,1 & 36,6 & 3,6 & 26,7 & 100,0 \\ \text { Diputado ...... } & 62,8 & 15,7 & 1,4 & 20,1 & 100,0 \\ \text { Arquitecto .... } & 64,2 & 13,0 & 0,6 & 22,2 & 100,0 \\ \text { Abogado...... } & 59,0 & 16,1 & 0,4 & 24,5 & 100,0 \\ \text { Profesor ...... } & 0,6 & 29,9 & 63,6 & 5,9 & 100,0 \\ \text { Agricultor..... } & 0,4 & 9,2 & 74,4 & 16,0 & 100,0\end{array}$

Fijándonos en la columna de opinión sobre sueldo "justo", obtendremos la siguiente jerarquía: $1 .^{\circ} \mathrm{Juez}$.

$$
2^{\circ} \text { Profesor. }
$$

Pero de estas tres profesiones tan sólo la primera, el porcentaje que opina que es "justo" es superior a las restantes opiniones. De esta forma debemos fijarnos más en las dos restantes opiniones.

La escala de opinión sobre el sueldo que aparece como "demasiado" es la siguiente: Médico $(66,6 \%)$, Arquitecto $(64,2 \%)$, Diputado (62,8\%), Empresario $(59,4 \%)$, Militar $(59,1 \%)$, Abogado (59\%) e Ingeniero $(54,3 \%)$. En todas estas profesiones aparecen porcentajes superiores a la mitad de los profesores encuestados. Esta opinión se completa con la jerarquía obtenida en la columna "poco". Así, pues, son estas profesiones, en ese orden, quienes se perciben como sobrerretribuidas.

El Juez aparece como única profesión con retribuciónes más justas en opinión de los maestros.

Las profesiones que obtienen mayor porcentaje de calificación de "poco" sueldo son las siguientes:

$$
\begin{aligned}
& 1 .^{\circ} \text { Agricultor }(74,4 \%) \\
& 2 .^{\circ} \text { Profesor }(63,6 \%) \\
& 3 .^{\circ} \text { Sacerdote }(58,0 \%)
\end{aligned}
$$

La propia profesión aparece reivindicada en cuanto a retribución. La mayor parte de los profesores opinan que ganan "poco". Además en comparación con otras profesiones se percibe claramente discriminada no en prestigio, sino en sueldo.

También hay un elevado porcentaje que no contesta, pero éste desciende notablemente cuando se trata de calificar la propia profesión.

En relación al sexo podemos señalar que el porcentaje de no contesta es muy elevado en las mujeres, llegando hasta el 33,1\% del total de mujeres encuestadas. Esto dificulta para analizar la interrelación entre dicha opinión y el sexo.

A pesar de esto podemos señalar:

- Interrelaciones con la opinión de sueldo "demasiado":

Hay mayor porcentaje de varones que de mujeres que opinan de esta forma en : Médico, Ingeniero, Empresario, Arquitecto. Así por ejemplo, hay un $20,1 \%$ más de varones que de mujeres que opinan que el sueldo de los Ingenieros es "demasiado alto".

- Interrelaciones con la opinión de sueldo "poco": 
Hay mayor porcentaje de varones que de mujeres que opinan que es poco en: Sacerdote, Agricultor y Profesor. Asi, por ejemplo, hay un $16,0 \%$ más de varones que de mujeres que opinan que el sueldo de los Profesores es "poco".

La edad interrelaciona con la opinión sobre los sueldos de diversas profesiones. Estas relaciones podemos situarlas así:

- Interrelación de la opinión de "demasiado" sueldo y edad.

A menor edad, mayor porcentaje de profesores que opinan que es "demasiado":

- Militar. Por ejemplo, hay un $17,6 \%$ más de varones entre 20 y 35 años que de varones entre 36 y 50 años que opinan que es un sueldo "demasiado" alto.

- Empresario. Por ejemplo, hay un $15,2 \%$ más de mujeres entre 20 y 35 años que de mujeres entre 36 y 50 que opinan que el sueldo de los empresarios es "demasiado" alto.

- Abogado. Por ejemplo, hay un 17,9\% más de mujeres entre 20 y 35 años que de mujeres entre 36 y 50 años que opinan que los abogados ganan "demasiado".

- Interrelación de la opinión de "poco" sueldo y la edad.

A menor edad, mayor porcentaje de profesores que opinan que es "poco":

- Profesor. Hay un 24,2\% más de varones entre 20 y 35 años que de varones mayores de 51 años que opinan que los profesores ganan "poco".

- Agricultor. Por ejemplo, hay un 10,5\% más de mujeres entre 20 y 35 años que de mujeres entre 36 y 50 años que piensan que los agricultores ganan "poco".

\section{4.- Capacitación profesional}

Recabamos la opinión de los profesores sobre la preparación de los diversos profesionales. Seguimos igual método que el referido en la pregunta anterior sobre el prestigio social.

Tabla IV. Opinion sobre la preparación de los profesionales.

Puntuacion

$\begin{array}{lccccc}\text { Profesión } & \text { ALTA } & \text { MEDIA } & \text { BAJA } & \text { No Contesta } & \text { TOTAL } \\ \text { Médico ........ } & 30,3 & 35,2 & 8,4 & 26,1 & 100,0 \\ \text { Ingeniero ..... } & 40,8 & 26,0 & 3,6 & 29,6 & 100,0 \\ \text { Militar ....... } & 26,3 & 28,8 & 15,3 & 29,6 & 100,0 \\ \text { Empresario ... } & 18,0 & 37,0 & 15,4 & 29,6 & 100,0\end{array}$




\begin{tabular}{|c|c|c|c|c|c|}
\hline 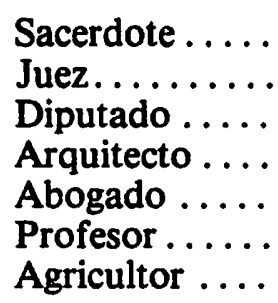 & $\begin{array}{l}35,4 \\
38,1 \\
12,9 \\
37,8 \\
29,8 \\
29,0 \\
25,2\end{array}$ & $\begin{array}{l}28,3 \\
26,7 \\
29,6 \\
27,9 \\
35,5 \\
38,6 \\
29,0\end{array}$ & $\begin{array}{r}8,7 \\
6,2 \\
27,9 \\
5,1 \\
5,4 \\
7,5 \\
16,9\end{array}$ & $\begin{array}{l}27,6 \\
29,0 \\
29,6 \\
29,2 \\
29,3 \\
24,9 \\
28,9\end{array}$ & $\begin{array}{l}100,0 \\
100,0 \\
100,0 \\
100,0 \\
100,0 \\
100,0 \\
100,0\end{array}$ \\
\hline
\end{tabular}

A la vista del cuadro anterior cabe mencionar la tasa muy alta de profesores que no contestan a dicha pregunta. La tasa es tan elevada que hace disminuir mucho la representatividad de los resultados y, por consiguiente, de las conclusiones. Aún asi analizamos la escala siguiente:

Pocas profesiones se ven con preparación baja. Los profesores tienden a evitar calificar con dicha puntuación, salvo en el ćaso de los diputados. De esta forma sólo podemos decir qué profesiones han sido menos vistas con alta o media preparación, y nunca podemos decir, salvo la excepción ya citada, que son vistas con baja preparación.

Se forma una jerarquía de profesiones según es vista su respectiva preparación por los profesores que aqui contestan. El primer lugar lo ocupa ingeniero le sigue juez y arquitecto. A continuación van: sacerdote, médico, abogado y profesor. La propia profesión es situada en un lugar bajo de la escala de preparación, además ocupa el lugar a partir del cual empiezan a emitirse opiniones significativas en el sentido de baja preparación. Es así que las profesiones vistas con menor preparación son: militar, agricultor, empresario y la única profesión que tiene más porcentaje en baja preparación que en alta es la de diputado, la que es vista con peor preparación.

Por tanto es poca la preparación que ven en la propia profesión al comparar con otras profesiones. Es notable sin embargo, el lugar que ocupan en esta escala de preparación y el lugar que ocupan en la anterior de prestigio algunas profesiones en relación al profesorado: los profesores se sitúan a sí mismos en la escala de prestigio por encima de sacerdote y abogado, mientras que en la de preparación se sitúan por debajo. Este diformismo puede observarse en el cuadro comparativo de las diferentes escalas.

La interrelación por sexo queda desdibujada porque la proporción de mujeres que no contestan es muy alta. Desde el $34 \%$ al $42 \%$ del total de mujeres encuestadas. Los varones, en mayor proporción que las mujeres, asignan una puntuación alta a las profesiones de ingeniero, juez y arquitecto. En cambio son más proclives que las mujeres, en dar puntuación baja a la preparación del diputado.

La edad también interrelaciona con las opiniones sobre la preparación. Así existe correlación en los siguientes casos:

Médico

Juez

Arquitecto

Abogado

Agricultor 
En éstas, a menor edad del profesor les atribuye mayor puntuación. Así, por ejemplo, hay un $9 \%$ más de profesores entre 20 y 35 años que de profesores entre 36 y 50 que les dan puntuación alta.

Escala de profesiones según prestigio, preparación y sueldo

$\begin{array}{lll}\begin{array}{l}\text { Prestigio } \\ \text { Médico }\end{array} & \begin{array}{l}\text { Preparacion } \\ \text { Ingeniero }\end{array} & \begin{array}{l}\text { Retribuciones } \\ \text { Médico }\end{array} \\ \text { Ingeniero } & \text { Juez } & \text { Arquitecto } \\ \text { Arquitecto } & \text { Arquitecto } & \text { Diputado } \\ \text { Juez } & \text { Sacerdote } & \text { Empresario } \\ \text { Profesor } & \text { Médico } & \text { Militar } \\ \text { Sacerdote } & \text { Abogado } & \text { Abogado } \\ \text { Abogado } & \text { Profesor } & \text { Ingeniero } \\ \text { Diputado } & \text { Militar } & \text { Juez } \\ \text { Empresario } & \text { Agricultor } & \text { Sacerdote } \\ \text { Agricultor } & \text { Empresario } & \text { Profesor } \\ \text { Militar } & \text { Diputado } & \text { Agricultor }\end{array}$

Para elaborar esta tabla hemos cogido y ordenado la columna de la opción "demasiado", en el caso del sueldo, y "alta" en el prestigio y preparación. Hay que anotar que la calificación de "demasiado" sueldo lleva connotaciónes de censura moral que se corresponden con el prestigio y la preparación o que no pueden corresponderse. Es decir, "demasiado" no tiene las mismas connotaciones que sueldo "alto".

Podíamos concluir nuestra reflexión anotando que los profesores rurales de E.G.B. en Castilla y León tienen una autoestima socioprofesional de infravaloración con el conjunto de otras profesiones equiparables, aunque en tendencia ascendente.

Aún reconociendo el valor relativo de las medidas de autoestima y autoprestigio comparativo, nuestra investigación confirma la hipótesis según la cual la condición social de maestro ha intentado escapar, más o menos parcialmente, a todo intento de valoración comparativa en el establecimiento de categorías profesionales. Se perciben, sin embargo, los caracteres de infravaloración comparativa. Por una parte, el salario -que significa el poder y el prestigio concedido al dinero- le ha colocado en una posición desfavorable. Por otra parte, el título - que simboliza el poder y el prestigio vinculado al saberle coloca en posiciones más halagadoras. Pero aunque el maestro está englobado en la categoría de "profesor", ésta es totalmente heterogénea, y el maestro ocupa en ella el último escalón jerárquico, con lo que también desde esta perspectiva se sitúa en una posición de infravaloración.

Actualmente, los maestros se encuentran en una situación incómoda. No se les reconoce ni la especificidad pedagógica, ni la posesión de un saber específico. Quieren, como otros profesionales, atribuirse uno de los grandes valores de nuestro tiempo: la especialización del saber. Sin embargo, su parcela de 
dominio específico y particular queda difusa y no privatizada: las ciencias de la educación. Se hallan así, en una posición de debilidad respecto a otros ensenantes y a otros profesionales.

Todo el interés y la sugestión de los maestros por adquirir diplomas o titulaciones complementarias, más que a la mejora de su capacitación docente, tienden a tratar de mejorar su imagen en relación a las categorías socioprofesionales que gozan de mayor prestigio.

\section{BIBLIOGRAFIA}

ABRAHAM, A.: El mundo interior del docente. Promoción cultural, Barcelona, 1975.

AGANZO, A.: "Experiencia de acción educativa en el mundo rural" en Documento Social, n. ${ }^{\circ} 49$ pág. $155-166$

AGANZO, A.: "Movimiento educativo rural: escuelas campesinas", en Documentación Social n. $0^{\circ} 49$, pág. $87-98$

BASTIDE, H.: Institutrice de Village. Mercure de France. 1970

BERGER, I.: Psicologia de los enseñantes. Tratado de Ciencias Pedagógicas. Vol. 9 págs. 147166. Oikos-Tau. Barcelona, 1974

BERGER, 1. y BENJAMIN, R.: L'univers des instituteurs. Ed. Minuit. Paris, 1964.

ELLIOT, P.: Sociologia de las profesiones. Ed. Tecnos. Madrid, 1977

GONZALEZ REGIDOR, J. y CARMENA LOPEZ, G.: La escuela en el medio rural. M.E.C., Madrid, 1984.

GRANDE RODRIGUEZ, M.: La escuela rural. Ed. Escuela popular. Granada, 1981.

GRANDE RODRIGUEZ, INFESTAS, A. y HERNANDEZ, J.M.: La educacion en Castilla y León. Ambito. Valladolid, 1984.

GIMENO SACRISTAN, J.: La formación del Profesor de E.G.B. Ministerio de Universidades e Investigación.

GOMEZ BARNUSELL, A.: El magisterio como profesión. Ed. Ariel, Barcelona, 1972.

GOBLE N. M. y PORTER, J.F.: La cambiante función del profesor. Ed. Narcea. Madrid. 1980.

I.N.E.M.: El prestigio Social de las ocupaciones en España, Madrid, 1978.

JACKSON, J.A.: Professions and Professionalisation, Cambridge, 1970.

LERENA, C.: El oficio de Maestro, en Sistema, Noviembre, 1982.

MASJOAN, J.: Els mestres a Catalunya. Ed. Nova Terra, Barcelona, 1974.

MOLLO, S. y OTROS.: "La representation de la condition du maitre dans la societe", en Enfance, n. ${ }^{\circ} 2-3$. Abril 1966, págs. 1-60.

MONCADA, A.: La profesion de enseñante, en Información Comercial Espantola, n. ${ }^{\circ} 537$, 1978.

NEIL, A.S.: Journal d'un instituteur de campagne. Ed. Payot, Paris, 1975.

ORDEN, A. de la: "Un problema inaplazable: la formación profesional del profesor" en Revista de Educación. Enero-Abril, 1982.

POSTIC, M.: Observación y formación de los profesores. Ed. Morata, Madrid, 1978. 